\title{
Rhabdomyomas and Tuberous sclerosis complex: our experience in 33 cases
}

\author{
Pietro Sciacca ${ }^{1 *}$, Valentina Giacchi ${ }^{2}$, Carmine Mattia ${ }^{3}$, Filippo Greco ${ }^{2}$, Pierluigi Smilari ${ }^{2}$, Pasqua Betta ${ }^{3}$ \\ and Giuseppe Distefano ${ }^{1}$
}

\begin{abstract}
Background: Rhabdomyomas are the most common type of cardiac tumors in children. Anatomically, they can be considered as hamartomas. They are usually randomly diagnosed antenatally or postnatally sometimes presenting in the neonatal period with haemodynamic compromise or severe arrhythmias although most neonatal cases remain asymptomatic. Typically rhabdomyomas are multiple lesions and usually regress spontaneously but are often associated with tuberous sclerosis complex (TSC), an autosomal dominant multisystem disorder caused by mutations in either of the two genes, TSC1 or TSC2. Diagnosis of tuberous sclerosis is usually made on clinical grounds and eventually confirmed by a genetic test by searching for TSC genes mutations.
\end{abstract}

Methods: We report our experience on 33 cases affected with rhabdomyomas and diagnosed from January 1989 to December 2012, focusing on the cardiac outcome and on association with the signs of tuberous sclerosis complex. We performed echocardiography using initially a Philips Sonos 2500 with a 7,5/5 probe and in the last 4 years a Philips IE33 with a S12-4 probe. We investigated the family history, brain, skin, kidney and retinal lesions, development of seizures, and neuropsychiatric disorders.

Results: At diagnosis we detected 205 masses, mostly localized in interventricular septum, right ventricle and left ventricle. Only in 4 babies (12\%) the presence of a mass caused a significant obstruction. A baby, with an enormous septal rhabdomyoma associated to multiple rhabdomyomas in both right and left ventricular walls died just after birth due to severe heart failure. During follow-up we observed a reduction of rhabdomyomas in terms of both number and size in all 32 surviving patients except in one child. Eight patients (24,2\%) had an arrhythmia and in 2 of these cases rhabdomyomas led to Wolf-Parkinson-White Syndrome. For all patients the arrhythmia spontaneously totally disappeared or was reduced gradually. With regarding to association with tuberous sclerosis, we diagnosed tuberous sclerosis clinically in 31 babies (93,9\%).

Conclusion: Rhabdobyomas are tumors with favorable prognosis because they frequently do not cause symptoms and they often regress in numbers and size. Nevertheless, due to frequent association with tuberous sclerosis complex and the resulting neurological impairment, the prognosis can result unfavorable.

Keywords: Cardiac masses, Rhabdomyomas, Tuberous sclerosis complex, Echocardiography

\footnotetext{
* Correspondence: pietrosciacca@hotmail.it

${ }^{1}$ Pediatric Cardiology, AOU Policlinico-Vittorio Emanuele, via Santa Sofia,

78 - 95123 Catania, Italy

Full list of author information is available at the end of the article
} 


\section{Background}

Rhabdomyomas are the most frequent cardiac tumors in children followed by fibromas (25\% to $30 \%)$ [1,2] and less commonly by myxoma, lipoma, teratoma, hemangioma, mesothelioma and Purkinje cell tumour. They are often associated to tuberous sclerosis complex (TSC) [3]. This is an autosomal dominant neurocutaneous disorder that can affect every organ of the body, most commonly the brain, kidney, heart, and lungs [4]. We report our experience with regard to 33 patients with multiple cardiac rhabdmiomata masses, focusing on cardiac outcome and on the association with tuberous sclerosis complex.

\section{Methods}

From January 1989 to December 2012 in the Pediatric Cardiology Unit of University of Catania echocardiography was performed in 14238 patients below twelve months of age using initially a Philips Sonos 2500 with a $7,5 / 5$ probe and in the last 4 years a Philips IE33 with a S12-4 probe.

Every patient if of age, or at least one parent or legal guardian if underage, gave their written informed consent before the patient's inclusion in the study. The study was conducted in accordance with the Helsinki Declaration, and the study protocol was approved by the (local) Ethics Committee of the Medical University of Catania.

Rhabdomyomas were identified in 33 cases $(0,23 \%)$. We identified all these patients (Table 1) from the database of our Department of Pediatrics. Clinical features of postnatal examinations were documented from pediatric records and echocardiographic images reviewed from the computer database. Data included age at diagnosis, clinical presentation, physical examination findings (cyanosis, heart murmur, arrhythmia, heart failure), electrocardiogram (ECG), 24-hour ECG recording results, initial and last echocardiography findings (number of rhabdomyomas, location, presence of inflow or outflow tract obstruction, myocardial dysfunction), indication for surgery, outcome (partial or total regression, residual tumors), and follow-up period.

Thirty one infants with diagnosis of Tuberous Sclerosis Complex were identified among those who were initially diagnosed having cardiac rhabdomyomas.

We assessed cerebral lesions and development of neurological and skin signs, and eventually kidney and eye involvement in all patients affected by tuberous sclerosis (Table 2). Diagnosis of TSC was based on clinical criteria established in 1998 by the US Tuberous Sclerosis Complex Association [5]. Genetic test for TSC is available on 3 patients (9\%) and all are positive.

Follow-up consisted of clinical case review as well as records of investigationswas: subsequent to the diagnosis, all babies were subjected to at least 18 months cardiac follow-up with ECG, color Doppler echocardiography and
Holter-ECG every six months. Regarding TSC, under the supervision of a pediatric neurologist, we performed skin and ophthalmological examinations, abdomen ultrasound examination, assessment of occurrence of seizures, brain MRI, aimed at highlighting the typical lesions of tuberous sclerosis complex, and evaluation of mental retardation, psychomotor delay or behavioral and language disorders (the follow-up was between six months and ten years).

\section{Results}

At diagnosis we detected 205 masses: $6(2,9 \%)$ in right atrium, $1(0,5 \%)$ in left atrium, $4(1,9 \%)$ close to valves, $16(7,8 \%)$ in the right infundibulum, $75(36,6 \%)$ in interventricular septum, 45 (22\%) in right ventricular wall, 58 $(28,3 \%)$ in left ventricular wall. In 10 cases $(30,3 \%)$ rhabdomyomas had been detected antenatally with fetal echocardiography and confirmed at birth. For the other patients, the indications to perform the first echocardiographic assessment were arrhythmias, such as, atrial and/or ventricular ectopic beats in 2 cases $(6,1 \%)$, in 1 case (3\%) the appearance of seizures as infantile spasms, in 2 cases $(6,1 \%)$ occurrence of cyanosis, in $11(33,3 \%)$ cases presence of a heart murmur, whereas 7 cases $(21,2 \%)$ were found during routine screening. The mean age in postnatally diagnosed patients was 74,6 days. In 31 patients $(94 \%)$ we detected multiple masses that led us to define these as rhabdomyomas. Only in 2 patients $(6,1 \%)$ we observed a single cardiac mass, one intramural in the interventricular septum and one protruding in right ventricular cavity respectively. Of these two, TSC was diagnosed only in one.

In 4 babies (12\%) the presence of a mass caused a significant obstruction and/or clinical signs of heart failure and/or cyanosis due to right or left ventricular outflow tract obstruction. Among these 4, in a newborn (3\%), with a diagnosis of cardiac masses in the fetal period, surgery to remove the mass was carried out because of severe signs of heart failure and cyanotic spells; a newborn (3\%), with antenatal diagnosis of an enormous septal rhabdomyoma associated to multiple rabdomyomas in both right and left ventricular wall, died soon after birth, due to a severe heart failure; whereas in the other 2 patients, with left and right ventricular obstruction respectively, obstructing rhabdomyomas decreased in terms of size without need of medication. Moreover, in another patient (3\%) echocardiography revealed only slight hypokinesia without need for medical or surgical therapy. The other 28 patients (85\%) did not show signs of hemodynamic impairment.

During follow-up we observed a reduction of rhabdomyomas in terms of both number and size in all 32 surviving patients except in one child. We detected 102 masses: 2 (2\%) in right atrium, none $(0 \%)$ in left atrium, 
Table 1 Clinical presentation - first cardiac medical examination

\begin{tabular}{|c|c|c|c|c|c|}
\hline $\begin{array}{l}\text { Case } \\
\mathrm{n}^{\circ}\end{array}$ & $\begin{array}{l}\text { Age at } \\
\text { diagnosis }\end{array}$ & $\begin{array}{l}\text { Clinical signs at first } \\
\text { examination }\end{array}$ & ECG and Holter-ECG & $\begin{array}{l}\text { Echocardiography at } \\
\text { diagnosis }\end{array}$ & $\begin{array}{l}\text { Cardiac } \\
\text { surgery }\end{array}$ \\
\hline \multirow[t]{2}{*}{1} & 2 months & Arrhythmia & Atrial and ventricular ectopic beats & Multiple rhabdomyomas & No \\
\hline & & & WPW Syndrome & & \\
\hline 2 & 1 month & Heart murmur & Normal & Multiple rhabdomyomas & No \\
\hline 3 & 2 months & Heart murmur & Widened QRS & Multiple rhabdomyomas & No \\
\hline 4 & Fetal & $\begin{array}{l}\text { No symptoms but RVOT } \\
\text { obstruction }\end{array}$ & Normal & Multiple rhabdomyomas & No \\
\hline \multirow[t]{2}{*}{5} & 1 day & Heart murmur & Normal & Multiple rhabdomyomas & No \\
\hline & & LV obstruction & & & \\
\hline 6 & 1 day & Heart murmur & Normal & Multiple rhabdomyomas & No \\
\hline 7 & Fetal & No symptoms & RV hypertrophy & Lobulated mass in LV & No \\
\hline 8 & Fetal & No symptoms & Normal & Multiple rhabdomyomas & No \\
\hline 9 & 1 month & Heart murmur & RV hypertrophy & Lobulated mass in IVS & No \\
\hline \multirow[t]{2}{*}{10} & 1 day & Cyanosis & Atrial and ventricular ectopic beats & Multiple rhabdomyomas & No \\
\hline & & LV obstrution & & & \\
\hline 11 & 2 months & No symptoms & Atrial ectopic beats & Multiple rhabdomyomas & No \\
\hline 12 & 1 months & No symptoms & RV overload signs & Multiple rhabdomyomas & No \\
\hline 13 & Fetal & Heart failure Cyanosis & Atrial and ventricular ectopic beats & Multiple rhabdomyomas & Yes \\
\hline 14 & 1 day & Heart murmur & Normal & Multiple rhabdomyomas & No \\
\hline \multirow[t]{2}{*}{15} & 7 months & Heart murmur & Atrial and ventricular ectopic beats & Multiple rhabdomyomas & No \\
\hline & & & WPW Syndrome & & \\
\hline 16 & 1 day & No symptoms & Incomplete right bundle branch block & Multiple rhabdomyomas & No \\
\hline 17 & 9 months & Heart murmur & Normal & Multiple rhabdomyomas & No \\
\hline 18 & 1 day & Arhythmia & Atrial and ventricular ectopic beats & Multiple rhabdomyomas & No \\
\hline 19 & Fetal & No symptoms (hypokinesia) & Normal & Multiple rhabdomyomas & No \\
\hline 20 & 4 months & Heart murmur & $\begin{array}{l}\text { Ventricular ectopic beats and RV overload } \\
\text { signs }\end{array}$ & Multiple rhabdomyomas & No \\
\hline \multirow[t]{2}{*}{21} & Fetal & No symptoms & WPW Syndrome & Multiple rhabdomyomas & No \\
\hline & & & IVS overload signs & & \\
\hline 22 & 3 days & Heart murmur & Normal & Multiple rhabdomyomas & No \\
\hline 23 & 11 months & No symptoms & RV overload signs & Multiple rhabdomyomas & No \\
\hline 24 & 5 months & No symptoms & Atrial and ventricular ectopic beats & Multiple rhabdomyomas & No \\
\hline 25 & 1 day & Heart murmur & Normal & Multiple rhabdomyomas & No \\
\hline 26 & 9 months & Seizures & Normal & Multiple rhabdomyomas & No \\
\hline 27 & Fetal & No symptoms & Normal & Multiple rhabdomyomas & No \\
\hline 28 & 2 months & No symptoms & Normal & Multiple rhabdomyomas & No \\
\hline 29 & Fetal & No symptoms & RV overload signs & Multiple rhabdomyomas & No \\
\hline 30 & Fetal & Heart murmur & Normal & Multiple rhabdomyomas & No \\
\hline 31 & 7 days & Heart murmur & Normal & Multiple rhabdomyomas & No \\
\hline 32 & 21 days & No symptoms & RV hypertrophy & Multiple rhabdomyomas & No \\
\hline 33 & Fetal & Heart murmur & Ventricular ectopic beats & Multiple rhabdomyomas & No \\
\hline
\end{tabular}

$2(2 \%)$ near the valves, $6(5,8 \%)$ in the infundibulum, 40 $(39,2 \%)$ in interventricular septum, $25(24,5 \%)$ in right ventricular wall, $27(26,5 \%)$ in left ventricular wall. In 8 cases $(24,2 \%)$ we found atrial and/or ventricular ectopic beats and in 2 of these cases rhabdomyomas led to Wolf-Parkinson-White Syndrome. For all patients, drug treatment was not believed necessary and the arrhythmia spontaneously healed or was gradually reduced. 
Table 2 General clinical presentation - heart, brain, skin, eye and kidney involvement - and family history

\begin{tabular}{|c|c|c|c|c|c|c|}
\hline $\begin{array}{l}\text { Case } \\
\mathrm{n}^{\circ}\end{array}$ & Cardiac signs & $\begin{array}{l}\text { Neurological } \\
\text { signs }\end{array}$ & MRI & Skin lesions & Others & Family history \\
\hline \multirow[t]{2}{*}{1} & WPW Syndrome & \multirow[t]{2}{*}{ West Syndrome } & \multirow[t]{2}{*}{ Cortical tubers } & $\begin{array}{l}\text { Hypomelanotic } \\
\text { maculae }\end{array}$ & \multirow[t]{2}{*}{$\begin{array}{l}\text { Retinal hamartoma } \\
\text { Renal angiomyolipoma }\end{array}$} & \multirow[t]{2}{*}{ Negative } \\
\hline & $\begin{array}{l}\text { Multiple } \\
\text { rhabdomyomas }\end{array}$ & & & Facial angiofibroma & & \\
\hline \multirow[t]{2}{*}{2} & Heart murmur & West syndrome & \multirow[t]{2}{*}{ Cortical tubers } & $\begin{array}{l}\text { Hypomelanotic } \\
\text { maculae }\end{array}$ & \multirow[t]{2}{*}{ No } & \multirow{2}{*}{$\begin{array}{l}\text { Sister with } \\
\text { language } \\
\text { disorders }\end{array}$} \\
\hline & $\begin{array}{l}\text { Multiple } \\
\text { rhabdomyomas }\end{array}$ & $\begin{array}{l}\text { Psychomotor } \\
\text { delay }\end{array}$ & & Facial angiofibroma & & \\
\hline \multirow[t]{3}{*}{3} & Heart murmur & No & \multirow{3}{*}{$\begin{array}{l}\text { Cerebral white matter radial } \\
\text { migration lines }\end{array}$} & \multirow{3}{*}{$\begin{array}{l}\text { Hypomelanotic } \\
\text { maculae }\end{array}$} & \multirow[t]{3}{*}{ Retinal hamartoma } & \multirow[t]{3}{*}{ Positive for TSC } \\
\hline & Widened QRS & & & & & \\
\hline & $\begin{array}{l}\text { Multiple } \\
\text { rhabdomyomas }\end{array}$ & & & & & \\
\hline \multirow[t]{2}{*}{4} & \multirow{2}{*}{$\begin{array}{l}\text { Multiple } \\
\text { rhabdomyomas }\end{array}$} & West Syndrome & \multirow[t]{2}{*}{ Cortical tubers } & \multirow{2}{*}{$\begin{array}{l}\text { Hypomelanotic } \\
\text { maculae }\end{array}$} & \multirow[t]{2}{*}{ Retinal hamartoma } & \multirow[t]{2}{*}{ Negative } \\
\hline & & Mental retardation & & & & \\
\hline \multirow[t]{3}{*}{5} & Heart murmur & No & \multirow[t]{3}{*}{ Normal } & \multirow[t]{3}{*}{ No } & \multirow[t]{3}{*}{ No } & \multirow[t]{3}{*}{ Negative } \\
\hline & Cyanosis & & & & & \\
\hline & $\begin{array}{l}\text { Multiple } \\
\text { rhabdomyomas }\end{array}$ & & & & & \\
\hline \multirow[t]{3}{*}{6} & Heart murmur & West syndrome & Cortical tubers & $\begin{array}{l}\text { Hypomelanotic } \\
\text { maculae }\end{array}$ & Retinal hamartoma & Negative \\
\hline & \multirow[t]{2}{*}{$\begin{array}{l}\text { Multiple } \\
\text { rhabdomyomas }\end{array}$} & $\begin{array}{l}\text { Drug resistant } \\
\text { epilepsy }\end{array}$ & Subependymal nodules & Facial angiofibroma & & \\
\hline & & $\begin{array}{l}\text { Psychomotor } \\
\text { delay }\end{array}$ & $\begin{array}{l}\text { Cerebral white matter radial } \\
\text { migration lines }\end{array}$ & Forehead plaque & & \\
\hline 7 & Lobulated mass in LV & West Syndrome & Not performed & $\begin{array}{l}\text { Hypomelanotic } \\
\text { maculae }\end{array}$ & No & Negative \\
\hline \multirow[t]{4}{*}{8} & \multirow[t]{4}{*}{$\begin{array}{l}\text { Multiple } \\
\text { rhabdomyomas }\end{array}$} & Partial seizures & Cortical tubers & $\begin{array}{l}\text { Hypomelanotic } \\
\text { maculae }\end{array}$ & \multirow[t]{4}{*}{ Retinal hamartoma } & Positive \\
\hline & & $\begin{array}{l}\text { Lennox-Gastaut } \\
\text { syndrome }\end{array}$ & Subependymal nodules & Facial angiofibroma & & \\
\hline & & Mental retardation & Subependymal giant cell & & & \\
\hline & & Behavior disorders & cytoma & & & \\
\hline 9 & $\begin{array}{l}\text { Lobulated mass in } \\
\text { IVS }\end{array}$ & No & Not performed & $\begin{array}{l}\text { Hypomelanotic } \\
\text { maculae }\end{array}$ & No & Negative \\
\hline 10 & Cyanosis & No & Not performed & No & No & Positive for TSC \\
\hline & LV obstruction & & & & & \\
\hline & $\begin{array}{l}\text { Multiple } \\
\text { rhabdomyomas }\end{array}$ & & & & & \\
\hline 11 & Atrial ectopic beats & West syndrome & Cortical tubers & Hypomelanotic & Renal cysts & Negative \\
\hline & & & Subependymal nodules & & & \\
\hline & $\begin{array}{l}\text { Multiple } \\
\text { rhabdomyomas }\end{array}$ & & $\begin{array}{l}\text { Cerebral white matter radial } \\
\text { migration lines }\end{array}$ & & & \\
\hline 12 & RV overload signs & West Syndrome & Cortical tubers & $\begin{array}{l}\text { Hypomelanotic } \\
\text { maculae }\end{array}$ & No & Negative \\
\hline & $\begin{array}{l}\text { Multiple } \\
\text { rhabdomyomas }\end{array}$ & & & Facial angiofibroma & & \\
\hline 13 & $\begin{array}{l}\text { Heart failure and } \\
\text { cyanosis }\end{array}$ & West syndrome & Subependymal nodules & $\begin{array}{l}\text { Hypomelanotic } \\
\text { maculae }\end{array}$ & No & Negative \\
\hline
\end{tabular}


Table 2 General clinical presentation - heart, brain, skin, eye and kidney involvement - and family history (Continued)

\begin{tabular}{|c|c|c|c|c|c|c|}
\hline & $\begin{array}{l}\text { Atrial and ventricular } \\
\text { ectopic beats }\end{array}$ & Behavior disorders & \multirow{2}{*}{$\begin{array}{l}\text { Subependymal giant cell } \\
\text { astrocytoma Cerebral white } \\
\text { matter radial migration lines }\end{array}$} & & & \\
\hline & $\begin{array}{l}\text { Multiple } \\
\text { rhabdomyomas }\end{array}$ & $\begin{array}{l}\text { Psychomotor } \\
\text { delay }\end{array}$ & & & & \\
\hline \multirow[t]{2}{*}{14} & Heart murmur & $\begin{array}{l}\text { Drug resistant } \\
\text { epilepsy }\end{array}$ & Cortical tubers & $\begin{array}{l}\text { Hypomelanotic } \\
\text { maculae }\end{array}$ & No & Negative \\
\hline & $\begin{array}{l}\text { Multiple } \\
\text { thabdomyomas }\end{array}$ & Mental retardation & Subependymal nodule & & & \\
\hline \multirow[t]{3}{*}{15} & Heart murmur & West syndrome & Cortical tubers & $\begin{array}{l}\text { Hypomelanotic } \\
\text { maculae }\end{array}$ & $\begin{array}{l}\text { Retinal hamartoma, } \\
\text { Renal angiomyolipoma }\end{array}$ & \multirow{2}{*}{$\begin{array}{l}\text { Mother with } \\
\text { hypomel. } \\
\text { macula }\end{array}$} \\
\hline & WPW Syndrome & $\begin{array}{l}\text { Behavior and } \\
\text { language } \\
\text { disorders }\end{array}$ & Subependymal nodule & Facial angiofibroma & & \\
\hline & $\begin{array}{l}\text { Multiple } \\
\text { rhabdomyomas }\end{array}$ & & $\begin{array}{l}\text { Cerebral white matter radial } \\
\text { migration lines }\end{array}$ & & & \\
\hline \multirow[t]{3}{*}{16} & \multirow{2}{*}{$\begin{array}{l}\text { Incomplete right } \\
\text { bundle branch block }\end{array}$} & \multirow[t]{3}{*}{ Partial seizures } & Cortical tubers & \multirow{3}{*}{$\begin{array}{l}\text { Hypomelanotic } \\
\text { maculae }\end{array}$} & \multirow[t]{3}{*}{ No } & \multirow[t]{3}{*}{ Negative } \\
\hline & & & Subependymal nodule & & & \\
\hline & $\begin{array}{l}\text { Multiple } \\
\text { rhabdomyomas }\end{array}$ & & & & & \\
\hline \multirow[t]{3}{*}{17} & Heart murmur & Partial seizures & Cortical tubers & $\begin{array}{l}\text { Hypomelanotic } \\
\text { maculae }\end{array}$ & No & Positive \\
\hline & \multirow[t]{2}{*}{$\begin{array}{l}\text { Multiple } \\
\text { rhabdomyomas }\end{array}$} & $\begin{array}{l}\text { Lennox-Gastaut } \\
\text { syndrome }\end{array}$ & & \multirow[t]{2}{*}{ Facial angiofibroma } & & \\
\hline & & Mental retardation & & & & \\
\hline \multirow[t]{3}{*}{18} & $\begin{array}{l}\text { Atrial and ventricular } \\
\text { ectopic beats }\end{array}$ & West Syndrome & Cortical tubers & $\begin{array}{l}\text { Hypomelanotic } \\
\text { maculae }\end{array}$ & Renal angiomyolipoma & Negative \\
\hline & \multirow{2}{*}{$\begin{array}{l}\text { Multiple } \\
\text { rhabdomyomas }\end{array}$} & & & Facial angiofibroma & & \\
\hline & & & & Forehead plaque & & \\
\hline \multirow[t]{3}{*}{19} & \multirow[t]{3}{*}{$\begin{array}{l}\text { Multiple } \\
\text { rhabdomyomas }\end{array}$} & \multirow[t]{3}{*}{ Partial seizures } & Cortical tubers & $\begin{array}{l}\text { Hypomelanotic } \\
\text { maculae }\end{array}$ & \multirow[t]{3}{*}{$\begin{array}{l}\text { Retinal hamartoma renal } \\
\text { angiomyolipoma }\end{array}$} & \multirow[t]{3}{*}{ Positive } \\
\hline & & & Subependymal nodules & Facial angiofibroma & & \\
\hline & & & $\begin{array}{l}\text { Cerebral white matter radial } \\
\text { migration lines }\end{array}$ & & & \\
\hline \multirow[t]{4}{*}{20} & Heart murmur & West syndrome & Cortical tubers & \multirow{4}{*}{$\begin{array}{l}\text { Hypomelanotic } \\
\text { maculae }\end{array}$} & \multirow[t]{4}{*}{ Renal cysts } & \multirow[t]{4}{*}{ Positive } \\
\hline & $\begin{array}{l}\text { Ventricular ectopic } \\
\text { beats }\end{array}$ & $\begin{array}{l}\text { Psychomotor } \\
\text { delay }\end{array}$ & Subependymal nodule & & & \\
\hline & RV overload signs & & Subependymal giant cell & & & \\
\hline & $\begin{array}{l}\text { Multiple } \\
\text { rhabdomyomas }\end{array}$ & & astrocytoma & & & \\
\hline \multirow[t]{2}{*}{21} & WPW Syndrome & Partial seizures & Cortical tubers & \multirow{2}{*}{$\begin{array}{l}\text { Hypomelanotic } \\
\text { maculae }\end{array}$} & \multirow[t]{2}{*}{ no } & \multirow[t]{2}{*}{ Negative } \\
\hline & $\begin{array}{l}\text { Multiple } \\
\text { rhabdomyomas }\end{array}$ & Behavior disorders & Subependymal nodule & & & \\
\hline \multirow[t]{2}{*}{22} & Heart murmur & \multirow[t]{2}{*}{ No } & Cerebral white matter radial & Hypomelanotic & no & Negative \\
\hline & $\begin{array}{l}\text { Multiple } \\
\text { rhabdomyomas }\end{array}$ & & & & & \\
\hline 23 & RV overload signs & $\begin{array}{l}\text { Drug resistant } \\
\text { epilepsy }\end{array}$ & $\begin{array}{l}\text { Cerebral white matter radial } \\
\text { migration lines }\end{array}$ & $\begin{array}{l}\text { Hypomelanotic } \\
\text { maculae }\end{array}$ & Retinal hamartoma & Negative \\
\hline & Multiple & Mental retardation & & Facial angiofibroma & & \\
\hline & nyomas & & & Forehead plaque & & \\
\hline 24 & $\begin{array}{l}\text { Atrial and ventricular } \\
\text { ectopic beats }\end{array}$ & West syndrome & Cortical tubers & $\begin{array}{l}\text { Hypomelanotic } \\
\text { maculae }\end{array}$ & No & Negative \\
\hline & $\begin{array}{l}\text { Multiple } \\
\text { rhabdomyomas }\end{array}$ & $\begin{array}{l}\text { Psychomotor } \\
\text { delay }\end{array}$ & Subependymal nodules & & & \\
\hline
\end{tabular}


Table 2 General clinical presentation - heart, brain, skin, eye and kidney involvement - and family history (Continued)

\begin{tabular}{|c|c|c|c|c|c|c|}
\hline \multirow[t]{2}{*}{$\overline{25}$} & Heart murmur & \multirow[t]{2}{*}{ No } & \multirow[t]{2}{*}{ Cortical tubers } & \multirow{2}{*}{$\begin{array}{l}\text { Hypomelanotic } \\
\text { maculae }\end{array}$} & \multirow[t]{2}{*}{ no } & \multirow[t]{2}{*}{ Negative } \\
\hline & $\begin{array}{l}\text { Multiple } \\
\text { rhabdomyomas }\end{array}$ & & & & & \\
\hline \multirow[t]{3}{*}{26} & \multirow{3}{*}{$\begin{array}{l}\text { Multiple } \\
\text { rhabdomyomas }\end{array}$} & \multirow[t]{3}{*}{ West syndrome } & Cortical tubers & \multirow{3}{*}{$\begin{array}{l}\text { Hypomelanotic } \\
\text { maculae Forehead } \\
\text { plaque }\end{array}$} & \multirow[t]{3}{*}{ Retinal hamartoma } & \multirow[t]{3}{*}{ Negative } \\
\hline & & & Subependymal nodules & & & \\
\hline & & & $\begin{array}{l}\text { Subependymal giant cell } \\
\text { astrocytoma }\end{array}$ & & & \\
\hline \multirow[t]{3}{*}{27} & \multirow[t]{3}{*}{$\begin{array}{l}\text { Multiple } \\
\text { rhabdomyomas }\end{array}$} & West syndrome & Cortical tubers & $\begin{array}{l}\text { Hypomelanotic } \\
\text { maculae }\end{array}$ & \multirow[t]{3}{*}{ Retinal hamartoma } & \multirow[t]{3}{*}{ Negative } \\
\hline & & $\begin{array}{l}\text { Drug resistant } \\
\text { epilepsy }\end{array}$ & Subependymal nodules & Facial angiofibroma & & \\
\hline & & Mental retardation & & Forehead plaque & & \\
\hline \multirow[t]{3}{*}{28} & \multirow[t]{3}{*}{$\begin{array}{l}\text { Multiple } \\
\text { rhabdomyomas }\end{array}$} & West syndrome & Cortical tubers & $\begin{array}{l}\text { Hypomelanotic } \\
\text { maculae }\end{array}$ & \multirow[t]{3}{*}{ Retinal hamartoma } & \multirow[t]{3}{*}{ Negative } \\
\hline & & \multirow[t]{2}{*}{ Behavior disorders } & Subependymal nodules & \multirow[t]{2}{*}{ Facial angiofibroma } & & \\
\hline & & & $\begin{array}{l}\text { Cerebral white matter radial } \\
\text { migration lines }\end{array}$ & & & \\
\hline 29 & $\begin{array}{l}\text { Multiple } \\
\text { rhabdomyomas }\end{array}$ & No & Cortical tubers & $\begin{array}{l}\text { Hypomelanotic } \\
\text { maculae }\end{array}$ & No & Positive \\
\hline \multirow[t]{2}{*}{30} & \multirow{2}{*}{$\begin{array}{l}\text { Multiple } \\
\text { rhabdomyomas }\end{array}$} & \multirow[t]{2}{*}{ West Syndrome } & Cortical tubers & \multirow{2}{*}{$\begin{array}{l}\text { Hypomelanotic } \\
\text { maculae }\end{array}$} & \multirow[t]{2}{*}{ No } & \multirow[t]{2}{*}{ Positive for TSC } \\
\hline & & & $\begin{array}{l}\text { Cerebral white matter radial } \\
\text { migration lines }\end{array}$ & & & \\
\hline \multirow[t]{2}{*}{31} & Heart murmur & $\begin{array}{l}\text { Drug resistant } \\
\text { epilepsy }\end{array}$ & Cortical tubers & \multirow[t]{2}{*}{$\begin{array}{l}\text { Hypomelanotic } \\
\text { maculae }\end{array}$} & Retinal hamartoma & \multirow[t]{2}{*}{ Negative } \\
\hline & $\begin{array}{l}\text { Multiple } \\
\text { rhabdomyomas }\end{array}$ & Mental retardation & Subependymal nodules & & Renal angiomyolipoma & \\
\hline \multirow[t]{3}{*}{32} & \multirow[t]{3}{*}{$\begin{array}{l}\text { Multiple } \\
\text { rhabdomyomas }\end{array}$} & West Syndrome & Cortical tubers & $\begin{array}{l}\text { Hypomelanotic } \\
\text { maculae }\end{array}$ & \multirow[t]{3}{*}{ No } & \multirow[t]{3}{*}{ Negative } \\
\hline & & Mental retardation & Subependymal nodules & Facial angiofibroma & & \\
\hline & & & $\begin{array}{l}\text { Cerebral white matter radial } \\
\text { migration lines }\end{array}$ & & & \\
\hline 33 & Heart murmur & $\begin{array}{l}\text { Lennox-Gastaut } \\
\text { syndrome }\end{array}$ & Cortical tubers & $\begin{array}{l}\text { Hypomelanotic } \\
\text { maculae }\end{array}$ & Retinal hamartoma & Negative \\
\hline & Multiple & Mental retardation & Subependymal nodules & Facial angiofibroma & & \\
\hline & rhabdomyomas & & $\begin{array}{l}\text { Cerebral white matter radial } \\
\text { migration lines }\end{array}$ & Forehead plaque & & \\
\hline
\end{tabular}

With regarding to association with tuberous sclerosis (Table 2), we diagnosed tuberous sclerosis clinically in 31 babies $(93,9 \%)$ and confirmed diagnosis in 3 patients (9\%) by genetic tests. Familial history of the disease was positive in 8 cases $(24,2 \%)$.

MRI of brain was performed in 30 children and revealed the characteristic lesions of tuberous sclerosis in 29 (96,6\%): cortical tubers in 24 patients (80\%), subependymal nodules in 16 (53,3\%), subependymal giant cell astrocytoma in $4(13,3 \%)$ and cerebral white matter radial migration lines in 12 (40\%). Often two or more lesions coexisted in the same patients.

Of 31 affected, 25 (80,6\%) developed seizures during follow-up and we cannot exclude symptoms in the future for the remaining. In particular, West Syndrome was present in 16 children (54,3\%), Lennox-Gastaut in 3 (9,6\%), partial seizures in $5(16,1 \%)$ and drug resistant epilepsy in 5 (16,1\%). Moreover mental retardation was present in 9 (29\%), psychomotor delay in $5(16,1 \%)$, behavior disorders in $5(16,1 \%)$ and language disorders in $1(3,2 \%)$.

With regrads to skin lesions, we noticed hypomelanotic macules in all 31 patients with tuberous sclerosis (100\%), facial angiofibroma in $14(45,1 \%)$ and forehead plaque in 6 $(19,3 \%)$. Concerning the involvement of other organs, we detected retinal hamartoma in $13(41,9 \%)$, renal angiomyolipoma in $5(16,1 \%)$ and renal cysts in $2(6,4 \%)$.

\section{Discussion}

Cardiac tumours are extremely rare in children (0.027 to $0.17 \%$ ) [6]. More than one-half of pediatric cardiac tumors 
are diagnosed within the twelve months of life and are diagnosed both in prenatal and postnatal period [7]. The vast majority of primary cardiac tumours in children are benign, whilst approximately $10 \%$ are malignant [8].

Rhabdomyomas are the most common cardiac tumours in children $(45 \%)[9,10]$.

Echocardiography has been estabilished as the primary diagnostic tool for the evaluation of cardiac tumors in children [11]. Rhabdomyomas appear on ultrasound as round, homogeneous, hyperechogenic, intramural or intracavitary masses, sometimes multiple [12], predominantly localized within the ventricles but can be observed in the atria or caval veins and may lead to obstruction of cardiac valves or inflow/outflow tracts. They are typically asymptomatic but may also cause atrial or ventricular arrhythmias, sinus node dysfunction, heart block and pre-excitation [2,9]. Surgical resection is not usually considered unless they cause severe intractable arrhythmias, valvular obstruction, or congestive heart failure [13]. In any case they are often difficult to remove completely, because they are usually located deep in the myocardium [14].

In our patients rhabdomyomas were mostly placed in the ventricles (94\%), but also in the right atrium $(2,9 \%)$, left atrium $(0,5 \%)$ and/or valves $(1,9 \%)$. Most masses led only to a heart murmur $(33,3 \%)$ or were identified incidentally $(21,2 \%)$ during echocardiography, but also were referred rarely due to cyanosis $(6,1 \%)$ or arrhythmias $(24,2 \%)$ that totally disappeared or gradually decreased over time. Only one case with signs of heart failure was subjected to surgical resection with good results. Nevertheless, another baby, with severe left ventricular outflow obstruction due to a giant septal rhabdomyomas (Figures 1, 2, 3), died soon after birth.
Congenital cardiac rhabdomyomas represent a condition of particular interest for the researcher due to spontaneous regression of the tumours that occurs in more than one-half of cases [7].

Jozwiak et al. reported that partial resolution of the cardiac rhabdomyomas was reported in $50 \%$ of cases and complete regression in $18 \%$ and added that these tumors have been reported to grow or to appear de novo in $4 \%$ of patients with tuberous sclerosis [15]. Smith et al. showed similar data: regression rates of $60 \%$ in preadolescent tuberous sclerosis patients and $18 \%$ in adult tuberous sclerosis patients [16,17].

In our experience we noticed involution of rhabdomyomas in all cases with reduction of number and size, with a decrease of all masses in $51,3 \%$, confirming indirectly the histological observations that these lesions regress.

To explain the involution tendency we refer to pathological anatomy: tumours consist of pathognomonic spider cells with centrally placed cytoplasm containing the nucleus and myofibrils radiating to the cell wall [18]. These tumours that seem to originate from embryonic myocytes, represent hamartomas of striated muscular fibers occurring solely in the heart [14].

Immunohistochemical immunoreactivity with ubiquitin, associated with the degradation of myoflaments, progression of cytoplasmic vacuolization, enlargement of glycogen vacuoles, apoptosis and myxoid degeneration are the events providing a plausible explanation for the spontaneous regression of rhabdomyoma [17]. In other words, the involution may be related to the inability of the tumours to divide while the heart chambers grow [19] and this consideration may indicate that some still incompletely identified factors, involved in homeostatic

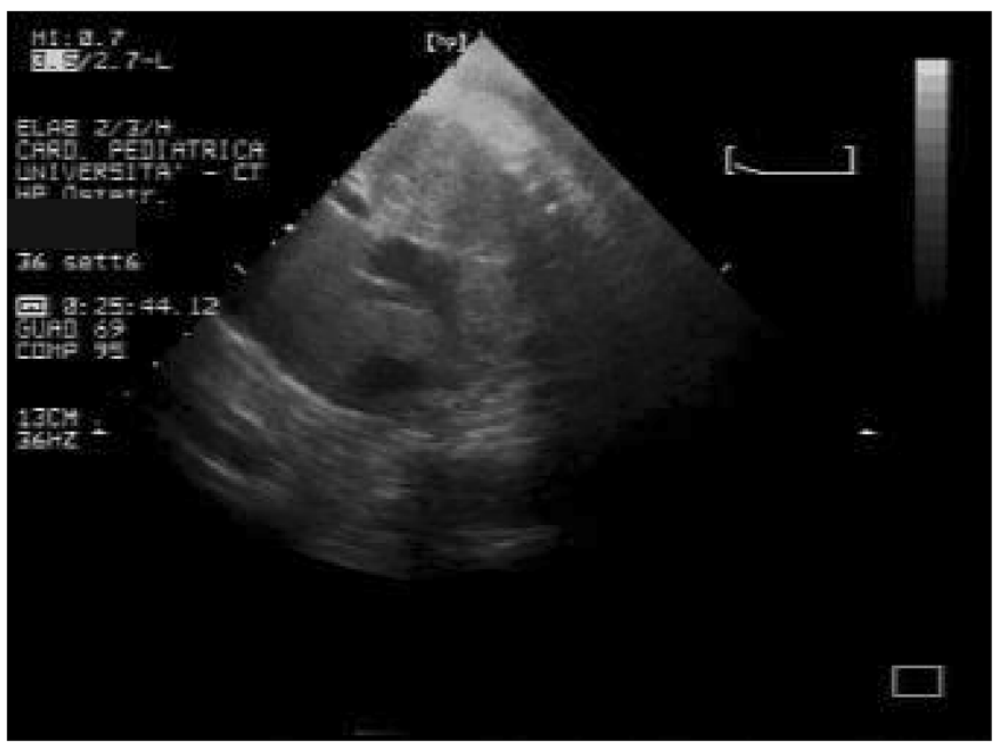

Figure 1 Giant fetal rhabdomyoma. 


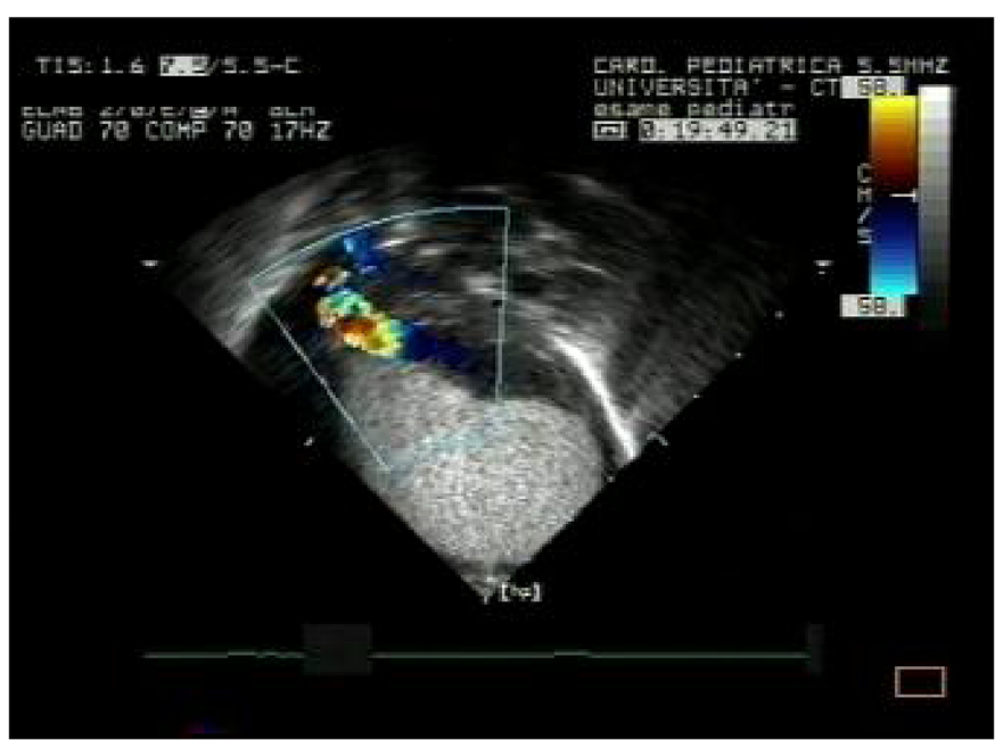

Figure 2 Giant rhabdomyoma in left ventricle.

regulation of cardiac biology, could lead to regression of the masses. Infact, after birth, rhabdomyoma cells lose their ability to divide and regression of the tumour in infancy is an expected outcome, regardless of size of the tumour [20-22]. Complete resolution of more than $80 \%$ of the tumours may occur during early childhood [23]. Regression may leave a scarred thin chamber wall [7].

The outcome of antenatally detected cardiac rhabdomyomas is also favorable. Once fetal somatic growth is completed, hamartomas lose their mitotic potential and undergo apoptosis [24]. The majority of tumours will regress towards the end of the third trimester although rarely some may continue to grow larger. Despite the expected shrinkage of these tumours, unexpected fetal loss may occur due to arrhythmias or obstruction of blood flow [20-22].

Rhabdomyomas can be sporadic but [2,9] in many cases they are associated with tuberous sclerosis complex (TSC) [25]. This is an inherited multiorgan disease with birth incidence of approximately 1 per 5,000 to 10,000 live births. It is an autosomal dominant neurocutaneous disorder characterized by tumor-like malformations that involve many organ systems, including the brain, heart, kidneys and skin [26].

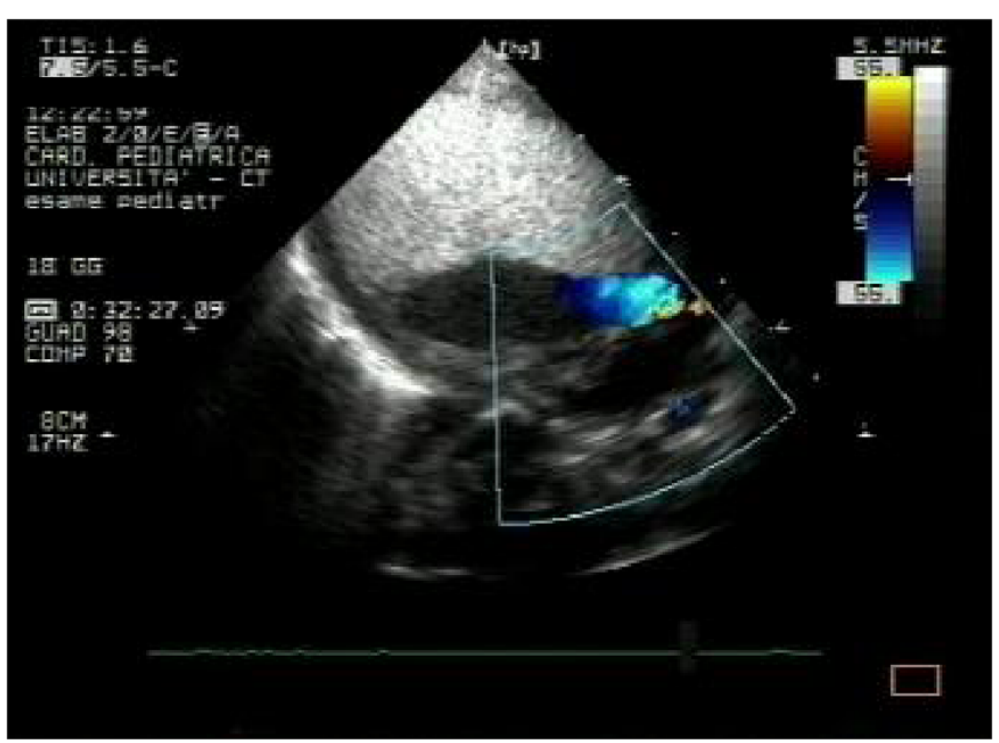

Figure 3 Giant rhabdomyoma - long axis. 
However, in up to $60 \%$ of cases, the disease is related to de novo mutations [27].

It is caused by mutations in either of the two genes, TSC1 or TSC2, which code for the proteins hamartin (chromosome 9q34) and tuberin (chromosome 16p13), respectively, that act as tumour-growth suppressors [28]. Hamartin and tuberin form a complex that activates the GTPase-activating protein Rheb to inhibit the mammalian target of rapamycin (mTOR). mTOR is a highly specific protein kinase that regulates protein synthesis, cellular metabolism, differentiation, growth, and migration. Constitutive activation of mTOR results in the abnormal cellular proliferation and differentiation responsible for the multiple hamartomatous growths throughout the central nervous system, lungs, heart, kidneys, eyes and skin [29].

In our experience rhabdomyomas were associated with tuberous sclerosis in a high number of cases $(93,9 \%)$ and we found a familial history positive for tuberous sclerosis in about a quarter of cases $(25,8 \%)$.

Our data mostly conform with the literature in that rhabdomyomas are strong predictors of TSC both when prenatally but especially when postnatally diagnosed [12,30,31].

It is reported that $60-80 \%$ of children affected by tuberous sclerosis have cardiac rhabdomyomas, whereas these tumors can be found in only around $20 \%$ of adults with tuberous sclerosis [19].

In our series almost all children had multiple rhabdomyomas (Figures 4 and 5) with diagnosis of TSC (96,7\%). Although the association of multiple cardiac rhabdomyomas with tuberous sclerosis has long been recognized, the association with a single rhabdomyoma is not clear.
However, in case of a solitary tumour a careful examination of cardiac chambers should be made in order not to miss smaller lesions elsewhere [32,33].

The diagnosis of TSC is made clinically. A clinical scoring system was developed that divides the diagnostic criteria for TSC into major and minor features. Single or multiple cardiac rhabdomyomas are considered a major feature.

However, the expression and the severity of the disease show substantial variation within, as well as between, families. The classical diagnostic triad of seizures, mental retardation, and facial angiofibromas occurs in fewer than half of the patients [5].

Neurologic manifestations are the most common; $90 \%$ of affected people experience seizures, and 30\% to $40 \%$ have mental retardation or autism [34,35].

Other signs of tuberous sclerosis include cutaneous lesions, such as, angiofibromas, shagreen patches and hypopigmented macules, brain lesions, such as, cortical/ subcortical tubers, subependymal nodules, subependymal giant cell astrocytomas and white matter lesions as well as renal and liver angiomyolipoma, retinal glial hamartomas and cysts in various locations, including the liver, kidneys and pancreas [36].

During follow-up our patients developed seizures in $80,6 \%$ of cases and we cannot exclude that the rest of the patients might develop them subsequently. West Syndrome represented the most frequent epilepsy occurring in $54,3 \%$ of the cases followed by partial seizures in $16,1 \%$, drug resistant epilepsy $(16,1 \%)$ and Lennox-Gastaut in $9,6 \%$. Other signs of neurological impairment were mental retardation $(29 \%)$, psychomotor delay $(16,1 \%)$, behavior

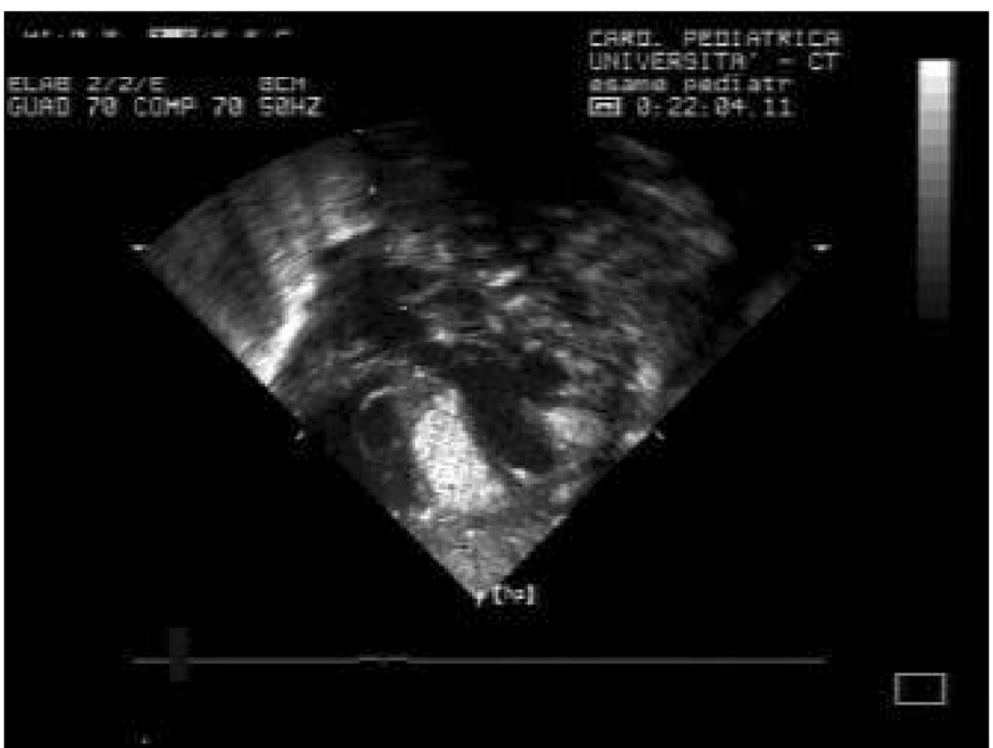

Figure 4 Multiple rhabdomyomas - 4 chambers. 


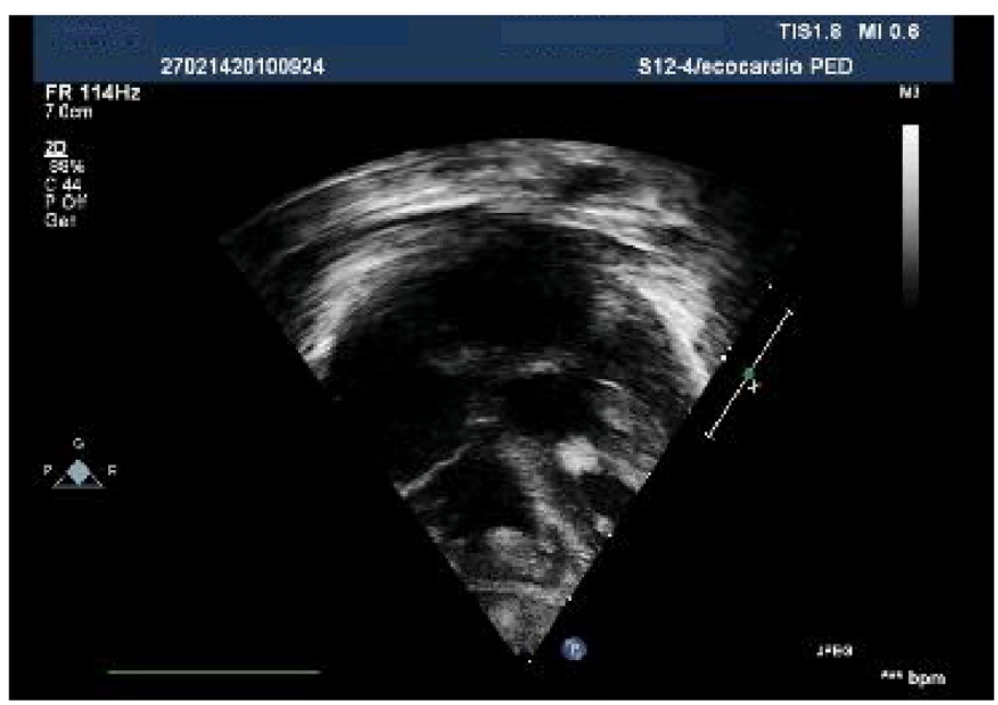

Figure 5 Multiple rhabdomyomas.

disorders $(16,1 \%)$ and language disorders in 1 (3,2\%). Regarding the brain lesions, they were present in almost all of these patients $(96,6 \%)$ and they mostly showed cortical tubers (80\%). Also the other lesions characteristic of TSC were present: subependymal nodules in few more than half of cases $(53,3 \%)$, cerebral white matter radial migration lines in less than half of cases $(40 \%)$ and, rarely, subependymal giant cell astrocytoma in only $13,3 \%$ of patients. Often two or more lesions coexisted in the same patient. Finally, about the other lesions of TSC, we found hypomelanotic macules in all, facial angiofibroma in $45,1 \%$ and shagreen patches in only $19,3 \%$. We detected retinal hamartoma in almost half of cases $(41,9 \%)$, renal angiomyolipoma in $16,1 \%$ and renal cysts in only $6,4 \%$. We cannot exclude that other lesions may develop over time.

Our data suggest that if the prognosis of rhabdomyomas in usually favorable, it is also true that in cases associated with tuberous sclerosis the general prognosis might be worsened by onset of seizures or lesions in other organs later in life.

\section{Conclusions}

Although there are no consistent guidelines, cardiac monitoring must be carried out for all tuberous sclerosis patients with rhabdomyomas, with serial echocardiography every 6 months and annual Holter monitoring, even if most patients are usually free from cardiac symptoms. Despite the potentially favourable prognosis of patients with cardiac rhabdomyomas, their presence should be sought (by echocardiography) in patients with tuberous sclerosis.

Regarding relation between rhabdomyomas and tuberous sclerosis we must consider that despite the potentially favourable cardiac evolution of patients with cardiac rhabdomyomas, their presence suggests a tuberous sclerosis with a neurological prognosis that is not related to the number or the dimensions of rhabdomyomas.

\section{Abbreviations}

TSC: Tuberous sclerosis complex.

\section{Competing interests}

The authors declare that they have no competing interests.

\section{Authors' contributions}

PS is the principal author and editor of this article. He has designed, written, reviewed the article and has given final approval of the version to be published. PS also provided the figures from his own collection. VG cowrited the article designed the tables, collected the data and revised the final manuscript and helped with the organisation of references. FG and PIS were involved in the neurological revising of data, PB CM and GD have been involved in interpretation of data. All authors read and approved the manuscript.

\section{Acknowledgements}

We acknowledge the excellent technical assistance of Nicola Bonanno of the Department of Pediatrics of University of Catania, Italy. We also acknowledge our secretary Concetta Scuderi for her collaboration in collecting data. All authors report no conflicts of interest to disclose.

\section{Work limitations}

About the association with tuberous sclerosis complex, we have not got the molecular analysis and/or to have its results for all patients. MRI is not performed in two patients. However we diagnosed tuberous sclerosis complex according clinical signs of disease.

\section{Author details}

${ }^{1}$ Pediatric Cardiology, AOU Policlinico-Vittorio Emanuele, via Santa Sofia, 78 - 95123 Catania, Italy. ${ }^{2}$ Department of Pediatrics, AOU Policlinico-Vittorio Emanuele, via Santa Sofia, 78 - 95123 Catania, Italy. ${ }^{3} \mathrm{NICU}, \mathrm{AOU}$ Policlinico-Vittorio Emanuele, via Santa Sofia, 78 - 95123 Catania, Italy.

Received: 20 October 2013 Accepted: 25 April 2014

Published: 9 May 2014 


\section{References}

1. Stratemann S, Dzurik T, Fish F, Parra D: Left ventricular cardiac fibroma in a child presenting with ventricular tachycardia. Pediatr Cardiol 2008, 29:223-226.

2. Burke A, Virmani R: Pediatric heart tumours. Cardiovasc Pathol 2008, 17:193-198.

3. Kocabaş A, Ekici F, Cetin II, Emir S, Demir HA, Arı ME, Değerliyurt A, Güven A: Cardiac rhabdomyomas associated with tuberous sclerosis complex in 11 children: presentation to outcome. Pediatr Hematol Oncol 2013, 30(2):71-79.

4. Singla S, Bansal M, Agarwal A: Mitral stenosis in tuberous sclerosis: a case of dystrophic calcification. J Postgrad Med 2012, 58(2):167.

5. Roach ES, Gomez MR, Northrup H: Tuberous sclerosis complex consensus conference: revised clinical diagnostic criteria. J Child Neurol 1998, 13(12):624-628.

6. Etuwewe B, John CM, Abdelaziz M: Asymptomatic cardiac rhabdomyoma in neonates: is surgery indicated? Images Paediatr Cardiol 2009, 11(2):1-8.

7. Myers KA, Wong KK, Tipple M, Sanatani S: Benign cardiac tumours, malignant arrhythmias. Can J Cardiol 2010, 26(2):58-61.

8. Uzun O, Wilson DG, Vujanic GM, Parsons JM, De Giovanni JV: Cardiac tumours in children. Orphanet J Rare Dis 2007, 2:11.

9. Freedom RM, Lee K, MacDonald C, Taylor G: Selected aspects of cardiac tumours in infancy and childhood. Pediatr Cardiol 2000, 21:299-316.

10. Becker AE: Primary heart tumours in the pediatric age group: A review of salient pathologic features relevant for clinicians. Pediatr Cardiol 2000, 21:317-323.

11. Marx GR, Moran AM: Cardiac tumors. In Moss and Adams' heart disease in infants, children and adolescents: including the fetus and young adults, Volume 2. 7th edition. Edited by Allen HD, Driscoll DJ, Shaddy RE, Feltes TF. Philadelphia: Lippincott Williams \& Wilkins; 2008:1479-1484.

12. Atalay S, Aypar E, Ucar T, Altug N, Deda G, Teber S, Tutar E: Fetal and neonatal cardiac rhabdomyomas: clinical presentation, outcome and association with tuberous sclerosis complex. Turk J Pediatr 2010, 52:481-487.

13. Venugopalan P, Babu JS, Al-Bulushi A: Right atrial rhabdomyoma acting as the substrate for Wolff-Parkinson-White syndrome in a 3-month-old infant. Acta Cardiol 2005, 60:543-545.

14. Di Liang C, Ko SF, Huang SC: Echocardiographic evaluation of cardiac rhabdomyoma in infants and children. J Clin Ultrasound 2000, 28:381-386.

15. Jóźwiak S, Kotulska K, Kasprzyk-Obara J, Domańska-Pakieła D, Tomyn-Drabik M, Roberts P, Kwiatkowski DJ: Clinical and genotype studies of cardiac tumors in 154 patients with tuberous sclerosis complex. Pediatrics 2006, 118:1146-1151.

16. Smith $\mathrm{HC}$, Watson $\mathrm{GH}$, Patel RG, Super M: Cardiac rhabdomyomata in tuberous sclerosis: their course and diagnostic value. Arch Dis Child 1989, 64:196-200

17. DiMario FJ Jr, Diana D, Leopold H, Chameides L: Evolution of cardiac rhabdomyoma in tuberous sclerosis complex. Clin Pediatr (Phila) 1996, 35:615-619.

18. Fenoglio JJ, McAllister HA, Ferrans VJ: Cardiac rhabdomyoma: a clinicopathologic and electron microscopic study. Am J Cardiol 1976, 38(2):241-251.

19. Benyounes N, Fohlen M, Devys JM, Delalande O, Moures JM, Cohen A: Cardiac rhabdomyomas in tuberous sclerosis patients: a case report and review of the literature. Arch Cardiovasc Dis 2012, 105(8-9):442-445.

20. Smythe JF, Dyck JD, Smallhorn JF, Freedom RM: Natural history of cardiac rhabdomyoma in infancy and childhood. Am J Cardiol 1990, 66:1247-1249.

21. Uzun O, McGawley G, Wharton GA: Multiple cardiac rhabdomyomas: tuberous sclerosis or not? Heart 1997, 77:388.

22. Paladini D, Palmieri S, Russo MG, Pacileo G: Cardiac multiple rhabdomyomatosis: prenatal diagnosis and natural history. Ultrasound Obstet Gynecol 1996, 7:84-85.

23. Bosi G, Linthermans JP, Pellegrino PA, Svaluto-Moreolo G, Vliers A: The natural history of cardiac rhabdomyoma with and without tuberous sclerosis. Acta Paediatr 1996, 85:928-931.

24. Satge D, De Geeter B: Rhabdomyomes cardiaques et apoptose:les régressions sont-elles controlées par l'organisme? Arch Mal Coeur Vaiss 1992, 85:603-608.

25. Lee KA, Won HS, Shim JY, Lee PR, Kim A: Molecular genetic, cardiac and neurodevelopmental findings in cases of prenatally diagnosed rhabdomyoma associated with tuberous sclerosis complex. Ultrasound Obstet Gynecol 2013, 41(3):306-311.

26. Adriaensen ME, Cramer MJ, Brouha ME, Schaefer-Prokop CM, Prokop M, Doevendans PA, Zonnenberg BA, Feringa HH: Echocardiographic screening results in patients with tuberous sclerosis complex. Tex Heart Inst J 2010, 37(3):280-283

27. Sampson JR, Scahill SJ, Stephenson JB, Mann L, Connor JM: Genetic aspects of tuberous sclerosis in the west of Scotland. J Med Genet 1989, 26:28-31.

28. Jabir S, Al-Hyassat S: Histological diagnosis of cardiac lipoma in an adult with tuberous sclerosis. BMJ Case Rep 2013, 3:2013.

29. Tiberio D, Franz DN, Phillips JR: Regression of a cardiac rhabdomyoma in a patient receiving everolimus. Pediatrics 2011, 127(5):1335-1337.

30. Bader RS, Chitayat D, Kelly E, Ryan G, Smallhorn JF, Toi A, Hornberger LK Fetal rhabdomyoma: 2003 prenatal diagnosis, clinical outcome, and incidence of associated tuberous sclerosis complex. J Pediatr 2003, 143(5):620-624.

31. Saada J, Hadj Rabia S, Fermont L, Le Bidois J, Bernardes LS, Martinovic J Sonigo P, Dumez Y, Bonnet D, Benachi A: Prenatal diagnosis of cardiac rhabdomyomas: incidence of associated cerebral lesions of tuberous sclerosis complex. Ultrasound Obstet Gynecol 2009, 34(2):155-159.

32. Nir A, Tajik AJ, Freeman WK, Seward JB, Offord KP, Edwards WD, Mair DD, Gomez MR: Tuberous sclerosis and cardiac rhabdomyoma. Am J Cardiol 1995, 76:419-421.

33. Webb DW, Thomas RD, Osborne JP: Cardiac rhabdomyomas and their association with tuberous sclerosis. Arch Dis Child 1993, 68:367-370.

34. Orlova KA, Crino PB: The tuberous sclerosis complex. Ann N Y Acad Sci 2010, 1184:87-105.

35. Crino PB, Nathanson KL, Henske EP: The tuberous sclerosis complex. N Engl J Med 2006, 355(13):1345-1356

36. Grajkowska W, Kotulska K, Jurkiewicz E: Matyja: Brain lesions in tuberous sclerosis complex. Review Folia Neuropathol 2010, 48(3):139-149.

doi:10.1186/1471-2261-14-66

Cite this article as: Sciacca et al:: Rhabdomyomas and Tuberous sclerosis complex: our experience in 33 cases. BMC Cardiovascular Disorders 2014 14:66

\section{Submit your next manuscript to BioMed Central and take full advantage of:}

- Convenient online submission

- Thorough peer review

- No space constraints or color figure charges

- Immediate publication on acceptance

- Inclusion in PubMed, CAS, Scopus and Google Scholar

- Research which is freely available for redistribution 